\title{
COATING THICKNESS INSPECTION WITH A FLASH LAMP AND AN INFRARED DETECTOR
}

\author{
${ }^{1}$ Lukáš MUZIKA, ${ }^{2}$ Michal ŠVANTNER, ${ }^{3}$ Šárka HOUDKOVÁ, ${ }^{4}$ Jiří MARTAN \\ ${ }^{1}$ University of West Bohemia, Plzeň, Czech Republic, EU, \\ 1'muzika@ntc.zcu.cz, ${ }^{2}$ msvatne@ntc.zcu.cz, ${ }^{3}$ houdkov@ntc.zcu.cz, ${ }^{4} j$ martan@ntc.zcu.cz
}

https://doi.org/10.37904/metal.2021.4174

\begin{abstract}
Thickness is one of important characteristics of coatings such as thermal barrier coatings, anticorrosion paints and others. The thickness of these coatings and its homogeneity can influence their properties and it is often measured quantity in industrial applications. Commonly used techniques are limited to a certain combination of coating and substrate. Thus, new techniques are still being developed. One of the new promising techniques for coating thickness estimation is flash pulse thermography. Its principle is in thermal excitation of a specimen with a flash lamp and capturing of thermal response with a high-speed infrared camera. Nowadays, the price of those cameras limits the usability of this technique only to scientific and high-tech purposes. This paper describes a possibility of replacing high-speed infrared cameras with infrared detectors, whose technical parameters are sufficient for this application and which price is significantly lower than the price of the suitable infrared cameras. Flash pulse thermographic inspection was applied for a thickness determination of thermally sprayed coatings using a high-speed infrared camera and a high-speed infrared detector. A comparison of both configurations showed benefits of replacing the high-speed infrared camera with the infrared detector. Advantages and disadvantages of both proposed procedures are discussed.
\end{abstract}

Keywords: Active thermography, coating thickness, thermally sprayed coatings, thermographic testing, thermographic inspection, infrared non-destructive testing, IRNDT

\section{INTRODUCTION}

Coatings are used in many different fields of industry to create surface layers which have different properties to the base material (substrate). Commonly coatings are used to protect base material against corrosion, heat, wear, acids and many more. For every purpose, different coating materials and coating technologies are used. One of the most important parameters of coated layer is its thickness. In case of insufficient thickness or its undesirable variation, the coating may not fulfill its purpose and can degrade more quickly. For those reasons, the coating thickness is often inspected.

Nowadays a lot of different destructive and non-destructive methods are used for coating thickness inspection [1]. Destructive tests (e.g. metallographic cross-section) provide clear information about coating thicknesses. Nonetheless, the problem with destructive tests is that they take a long time to perform, are expansive and the tested object is not further usable. Therefore, nondestructive tests are preferred if possible. Many different non-destructive testing techniques were developed (e.g. eddy current methods, ultrasonic methods, electromagnetic methods) and are used on day to day basis. Each technique is mostly usable only for a limited number of materials due to the physical principle of the testing method. Development of a universal fast method for coating thickness estimation could save costs and improve quality inspection of coated parts.

Flash pulse thermography is a promising alternative method for coatings thickness measurement [2], [3]. The principle of flash pulse thermography is thermal excitation of a tested object by one very short pulse ( $<10 \mathrm{~ms})$ and recording of thermal response by an infrared camera. The response may show changes in heat transfer, which are caused by different thermal properties, which can be caused by thickness differences. The response 
of the flash pulse thermography measurement is a sequence of images in the infrared (IR) spectrum (video in IR). Thickness can be then obtained by advanced processing of the flash-pulse thermography measurement response. More about the processing for thickness estimation can be found in [2] or [4].

Several articles showed the feasibility of flash pulse thermography for coating thickness inspection [2], [3], [4], [5]. Nonetheless, the cost of a necessary equipment is quite high, especially due to the need of a highspeed/high-sensitivity infrared camera. It limits widespread usage of this method for a common use. This disadvantage could be solved by replacing of an IR camera with an IR detector. Comparison of both solutions is analyzed and described in this paper.

\section{EXPERIMENT}

Experiments were performed on a sample made by Twin Wire Arc Spraying (TWAS) technology. The substrate was $25 \times 80 \mathrm{~mm}$ plate made of $5 \mathrm{~mm}$ thick S235 construction steel. The coating was from $\mathrm{Cr} 13 \% \mathrm{Fe}$. It was sprayed on the substrate in a way to create 4 zones (labeled A1-A4) with different coating thicknesses. The thickness of the zones A1, A2, A3 and A4 was 220, 380, 490 and $590 \mu \mathrm{m}$. Visualization of the tested specimen is in (Figure $1 \mathrm{a}$ ). The cross-section of the specimen at a substrate-coating interface is in (Figure $1 \mathbf{b}$ ).

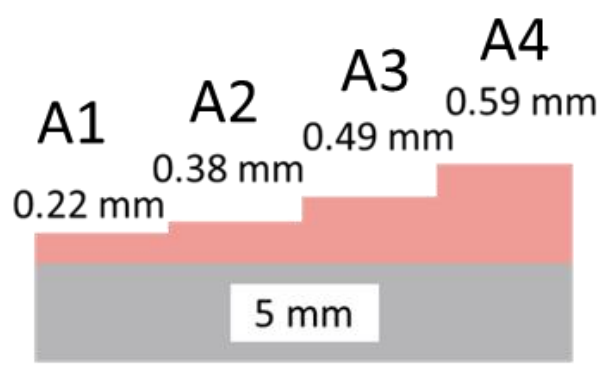

a)

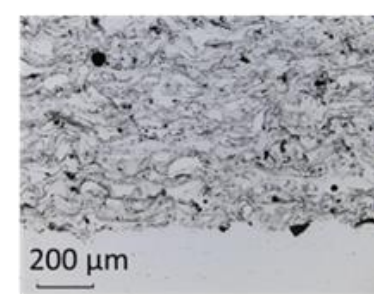

b)

Figure 1 Specimen a) visualization, b) cross-section

The specimen was measured in 2 configurations. In both cases a flash lamp Hensel 6000 EH Pro was used as an excitation source. Covering glass was placed before the flash lamp to suppress the afterglow effect. The lamp was placed vertically to the specimen at a distance of $40 \mathrm{~cm}$.

Two measurement configurations were used. In the configuration 1, the measurement was performed with IR camera FLIR SC7650 with NETD $20 \mathrm{mK}$ and integration time $1.4 \mathrm{~ms}$. The acquisition frequency was $250 \mathrm{~Hz}$. The resolution of the recording was $320 \times 256$ pixels. The camera was at the distance of $45 \mathrm{~cm}$ from the specimen. In the configuration 2, the measurement was performed with an IR detector VIGO PVI-4TE-10.61X1. Data from the detector was recorded by an oscilloscope Hameg HMO1022. The acquisition frequency was $1 \mathrm{kHz}$. The IR detector with Germanium optic was at the distance of $7 \mathrm{~cm}$ from the specimen.

\section{RESULTS}

Time series data measured by the IR camera and IR detector are in (Figure 2). Data from the IR detector were filtered to suppress noise with Savitzky-Golay filter. Time series recorded by both devices show a similar trend. Zones of smaller coating thickness ( $\mathrm{A} 1$ is the smallest thickness) cool down faster compared to the thickest coating (A4). This is caused by the thermal properties of both coating and substrate. In this case, the coating has smaller thermal conductivity and diffusivity than the substrate. It should be pointed out that properties of the coating are not influenced just by a material composition but also by spraying technology (e.g. porosity can influence thermal properties). 


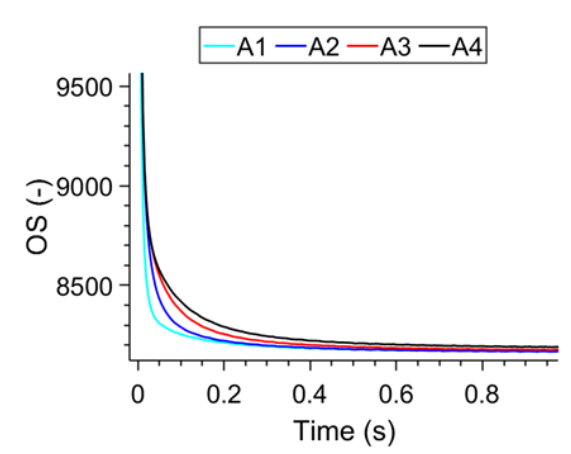

a)

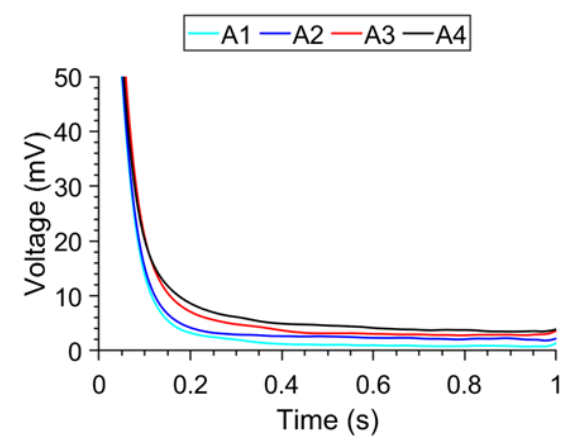

b)

Figure 2 Measurement response a) IR camera, b) IR detector

Differences between time series curves are in general too small to distinguish small differences in coating thickness. It is also difficult to use these data for a quantitative evaluation of the differences. Thus, advanced post-processing techniques were used on both datasets. Fast Fourier transform - phase [5] and P-function algorithm [6], [7]. Data obtained from those processing techniques can be used for functional correlation between thickness and processed data. In this case it would lead to functional correlation between phase and thickness (for Fast Fourier transform) and between characteristic time (P-function) and thickness.

Results of processed data (Phases and/or P-function characteristic times) are presented in (Table 1). It can be seen that the results of the IR camera measurement can be correlated with coating thicknesses. However, a clear correlation between the IR detector results and the thickness was not observed neither in the case of Phase nor P-function evaluation. The phase falls with the increasing thickness of the coating for the range A1A2. However, this trend is disrupted by the A3-A4, where the phase increases. A similar trend disruption can be found for P-function, where characteristic time increases for A1-A3 but decreases for A4.

Table 1 Results of advanced processing algorithms

\begin{tabular}{|c|c|c|c|c|c|}
\cline { 3 - 6 } \multicolumn{2}{c|}{} & A1 & A2 & A3 & A4 \\
\hline \multirow{3}{*}{ Camera } & Phase $(\mathrm{rad})$ & -0.39 & -0.43 & -0.54 & -0.59 \\
\cline { 2 - 6 } & P-function $(\mathrm{t})$ & 0.09 & 0.24 & 0.44 & 0.59 \\
\hline \multirow{3}{*}{ Detector } & Phase $(\mathrm{rad})$ & -0.24 & -0.44 & -0.36 & -0.29 \\
\cline { 2 - 6 } & P-function $(\mathrm{t})$ & 0.27 & 0.53 & 0.81 & 0.34 \\
\hline
\end{tabular}

The difference was caused due to different measurement units. Both IR camera and the IR detector measure radiation flux [8]. The output of the IR camera is temperature or radiation intensity. The conversion between the input radiation and the outputs is not linear and it is provided by the IR camera hardware and software including a calibration of the device. On the other hand, the output of the IR detector is raw electrical signal without any conversion procedures. Subsequent conversion should follow when a standard advanced processing is used.

It is evident from the time series dependences in (Figure 2) that the trends and difference between the individual coatings measured by the IR camera and IR detector are similar. However, it was shown that postprocessing procedures standardly used for the evaluation of IR camera flash-pulse measurement cannot be used for IR detectors without additional processing of their output signal. This shows that it would be possible to detect differences in coating thicknesses. Nonetheless for usage of flash pulse thermography algorithms, volts value should be converted to temperature. 


\section{CONCLUSION}

It can be concluded that the IR detector could be used as a replacement for the IR camera for coating thickness inspection with flash pulse thermography measurement, but additional calibration steps must be performed. Same trends were distinguishable on unprocessed datasets obtained from IR detector and IR camera.

The biggest disadvantage of an IR detector compared to an IR camera is that it measures just from one point. Thereby it is possible to obtain surface distribution of thickness only by the IR camera. In contrast, the IR detector provides a point measurement only. However, the advantage of the IR detector is its much lower price compared to high-speed and high-sensitivity IR cameras.

\section{ACKNOWLEDGEMENTS}

This The work has been supported by project ERDF "LABIR-PAV / Pre-application research of infrared technologies" (No. CZ.02.1.01/0.0/0.0/18_069/0010018) and project SGS-2019-008

\section{REFERENCES}

[1] GIURLANI, W., BERRETTI, E., INNOCENTI, M., LAVACCHI, A. Measuring the thickness of metal coatings: A review of the methods. Coatings. [online]. 2020, vol. 10, pp. 1-36. Available from:

https://doi.org/10.3390/coatings10121211.

[2] SHRESTHA, R., KIM, W. Evaluation of coating thickness by thermal wave imaging: A comparative study of pulsed and lock-in infrared thermography - Part II: Experimental investigation. Infrared Phys. Technol. [online]. 2018, vol. 92, pp. 24-29. Available from: https://doi.org/10.1016/j.infrared.2018.05.001.

[3] MUZIKA, L., ŠVANTNER, M., HOUDKOVÁ, Š., ŠULCOVÁ, P. Application of flash-pulse thermography methods for quantitative thickness inspection of coatings made by different thermal spraying technologies. Surf. Coatings Technol. [online]. 2021, vol. 406. Available from: https://doi.org/10.1016/j.surfcoat.2020.126748.

[4] ALTENBURG, S.J., KRANKENHAGEN, R., BAVENDIEK, F. Thickness determination of polymeric multilayer surface protection systems for concrete by means of pulse thermography. AIP Conf. Proc. [online]. 2017, vol. 1806. Available from: https://doi.org/10.1063/1.4974669.

[5] SHRESTHA, R., CHUNG, Y., KIM, W. Thermal Behavior Variations in Coating Thickness Using Pulse Phase Thermography. J. Korean Soc. Nondestruct. Test. [online]. 2017, vol. 36, pp 259-265. Available from: https://doi.org/10.7779/jksnt.2016.36.4.259.

[6] MUZIKA, L., ŠVANTNER, M., MOSKAL, D. Time transformation method for pulse thermography. In: Metal 2019 28th Int. Conf. Metall. Mater. Conf. Proc. Brno: Tanger, 2019.

[7] MUZIKA, L., ŠVANTNER, M., MOSKAL, D. Experimental investigation of a time-power transformation method for flash-pulse thermographic testing, Appl. Opt. [online]. 2020, vol. 59, pp. E89-E96. Available from: https://doi.org/10.1364/AO.388437.

[8] BUDZIER, H., GERLACH, G. Calibration of uncooled thermal infrared cameras. J. Sensors Sens. Syst. [online]. 2015, vol. 4, pp. 187-197. Available from: https://doi.org/10.5194/jsss-4-187-2015. 